\title{
MEG/STRD Modeling of Optical Antenna Sensors as Micro Rectangular Apertures
}

\author{
${ }^{1}$ Alessandro Massaro, ${ }^{2}$ Fabrizio Spano, ${ }^{4}$ Athanassia \\ Athanassiou \\ Center for Bio-Molecular Nanotechnologies (CBN) of IIT, \\ via Barsanti 1, 73010, Arnesano (LE), Italy.
}

\author{
${ }^{3}$ Roberto Cingolani \\ Italian Institute of Technology (IIT), Via Morego 30, 16163, \\ Genova, Italy.
}

\begin{abstract}
In this paper we present a novel model for the design of micrometric optical antennas. The accuracy of the model allows to evaluate the near field generated by a micrometric aperture in parallel-plate waveguides. The model starts from the equivalent source distribution of magnetic current excitation of a rectangular aperture and it proceeds by employing the multipole expansion of the Green's function (MEG) applied to the aperture, including the analysis of the electromagnetic field in proximity of metallic discontinuities. The presented method introduces the novel principle of simultaneous transverse resonance diffraction (STRD) which determines the order of singularity for different edges at optical frequencies. The approach is suitable for inclusion in standard electromagnetic (EM) simulators.
\end{abstract}

Keywords- MEG Modeling; Optical Antenna; Optical Sensor.

\section{INTRODUCTION}

Optical antennas are an emerging concept in physical optics. Similar to radiowave and microwave antennas, their purpose is to convert the energy of free propagating radiation to localized energy, and vice versa. In optical science and engineering, light is commonly controlled by redirecting the wave fronts of propagating radiation by means of lenses, mirrors, and diffractive elements. This type of manipulation relies on the wave nature of electromagnetic fields and is therefore not amenable to controlling fields on the subwavelength scale. In contrast, radiowave and microwave technology predominantly makes use of antennas to manipulate electromagnetic fields, controlling them on the subwavelength scale and interfacing efficiently between propagating radiation and localized fields. While antennas are a key enabling technology for devices like cellular phones and televisions using electromagnetic radiation in the radiowave or microwave regime, their optical analog is basically nonexistent in today's technology. However, recent research in nano-optics and plasmonics has generated considerable interest in the optical antenna concept, and several studies are currently focused on how to translate established radiowave and microwave antenna theories into the optical frequency regime. The micro-nano scale of antennas requires an accurate modeling especially for near field applications. The traditional numerical approaches such as finite element method (FEM), and finite difference time domain (FDTD) technique require
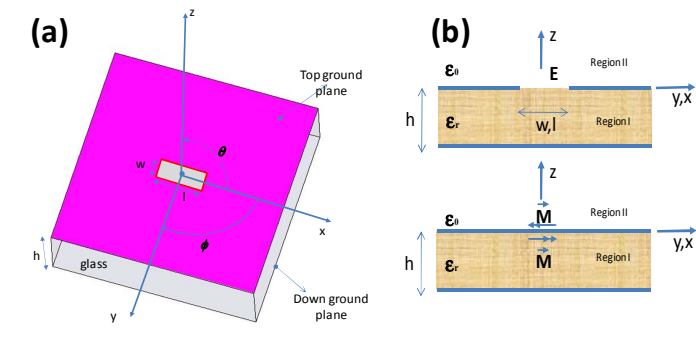

(c)

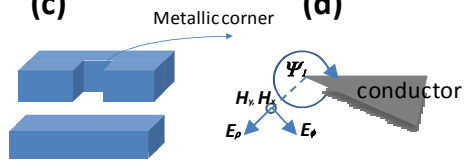

(e)

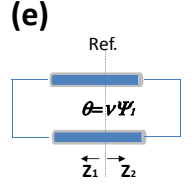

Fig. 1. (a) Optical antenna as micrometric aperture in parallel-plate waveguide. The aperture is in the top ground plane. (b) Cross section ( $x-z$ or $y-z$ plane) of the waveguide of Fig. 1: original electromagnetic problem (above) and equivalent problem (below). (c) Metallic corner of a slot aperture in parallelplate waveguide. (d) Electromagnetic field configuration for a propagating $\mathrm{TM}_{\mathrm{zn}}$ mode. (e) STRD transmission line modelling of the metallic corner.

high computational cost for the evaluation of the radiated field in proximity of a dielectric or metallic discontinuity. For this purpose we use the new approach of multipole expansion of the Green's function (MEG) based on the novel principle of simultaneous transverse resonance diffraction (STRD) [10]. The new approach was previously applied for the near field evaluation of three dimensional (3D) dielectric corner by showing a good convergent solution with a low computational cost with a reduction of the computational time of 15/12 times if compared with the traditional numerical methods [1]-[2]. For the first time we apply the MGE method to a particular class of antennas obtained as slot apertures in parallel-plate waveguides [3],[4]. The use parallel-plate waveguides in optics presents various advantages such as easy fabrication process (deposition of metallic layers on both side of a standard glass for chemical sample), the use of simplified experimental setups, and, the applicability in a large number of applications for sensing wireless detection systems especially for robotics and biomedical sensing. Moreover, the use of micrometric apertures on the waveguides allows to use the device in small spaces useful for implanted systems and aerospace wireless 
applications. However, by considering high frequencies in the optical frequency band the modeling of the radiation field coming from these apertures becomes difficult to improve. For this purpose we propose in this work the MEG/STRD approach which evaluates the radiation field, generated by a micromectric slot aperture in parallel-plate waveguides.

\section{STRD/MEG RESULTS}

The Fig. 1 shows the basic structure of an optical slot antennas in a parallel-plate configuration. The substrate (glass) of thickness $h$ has relative permittivity $\mathcal{E}_{r}$. In order to analyze the effect of parallel-plate waveguide on the optical radiation we consider the cross section reported in Fig. 1 (a): a guided mode propagates along the longitudinal direction, when it arrives at the aperture position will generate the near field as evanescent field due to the diffraction of the metallic discontinuity. Because of the equivalence principle, the geometry in Fig. 1 (b). In the region above (region I) and in the region below (region II) the top ground plane the $y, x$-directed magnetic field at $z=0$ excited by a parallel magnetic current $M_{y, x}$ are expressed in terms of the Green's function $\mathrm{G}^{\mathrm{I}, \mathrm{II}} \mathrm{y,x}$ :

$$
H_{y, x}^{(I, I I)}=\iint_{A} G_{y, x}^{(I, I I)}\left(x-x^{\prime}\right)\left(y-y^{\prime}\right) M_{y, x}^{(I, I I)}\left(x^{\prime}, y^{\prime}\right) d x^{\prime} d y^{\prime}
$$

where $A$ indicates the of the surface of the aperture, and the Green' function is defined by the following multipole expansion [1]-[2]

$$
G\left(\mathbf{r}, \mathbf{r}^{\prime}\right)=\sum_{s, m} g_{s}\left(r, r^{\prime}\right) Y_{s, m}^{*}\left(\theta^{\prime}, \varphi^{\prime}\right) Y_{s, m}(\theta, \varphi)
$$

The STRD/MEG approach considers the modeling of the rectangular aperture as illustrated in Fig. 1 (c), (d), (e) [1]-[2]. This modeling allows to implement the equivalent magnetic current of the function (1) (see Fig. 2 where is reported $M_{x, y}$ versus the working wavelength for different aperture sizes.) We observe from Fig. 2 that for a fixed $l$ the amplitude of the function $M_{y, x}$ increases by considering lower $w$ values. For a parametric analysis, we then fix the working wavelength at $\lambda_{0}=1.55 \mu \mathrm{m}$ (useful wavelength for telecommunication systems) by achieving the 3D plot of Fig. 3 where is reported the equivalent magnetic current distribution of a micrometric slot aperture versus both the parameters $l$ and $w$. We consider in our analysis metallic discontinuities with $\Psi_{1}=3 / 2 \pi$ and plane wave as source by calculating the radiation pattern reported in Fig. 4. We observe that the apertures can be fabricated by laser texturing technique (see Fig. 5).

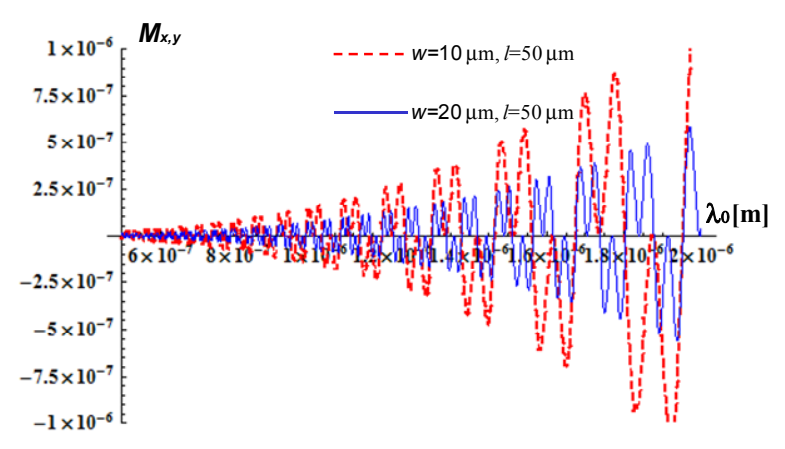

Fig. 2 Values of the equivalent magnetic current $M_{y, x}$ in arbitrary units versus the working wavelength. The analysis refers to the fundamental TEM mode.

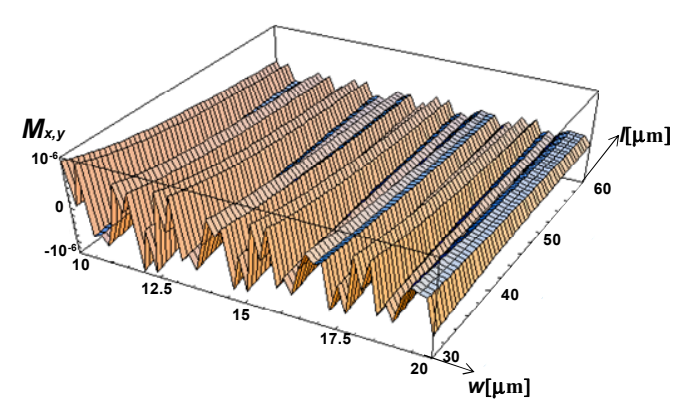

Fig. 3. Values of the equivalent magnetic current $M_{y, x}$ function in arbitrary units of equation (5) versus $l$ and $w$. The 3D plot is referred to the fundamental mode at $\lambda_{0}=1.55 \mu \mathrm{m}$. The analysis refers to the fundamental TEM mode.

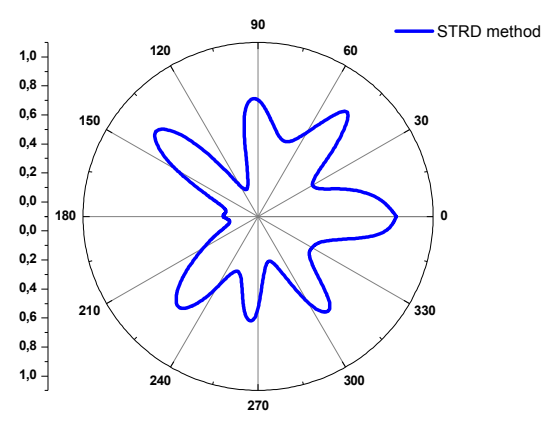

Fig. 4. STRD/MEG radiation pattern versus $\phi$ for $\theta=10 \mathrm{deg}$. The simulated slot aperture is characterized by $w=10 \mu \mathrm{m}$ and $l=30 \mu \mathrm{m}$ and $\lambda_{0}=1.55 \mu \mathrm{m}$.

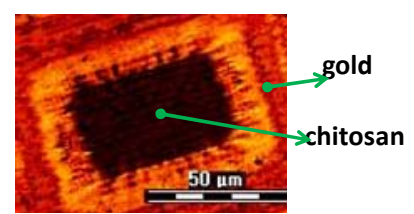

Fig. 5. Technology: example of micrometric rectangular aperture obtained by laser texturing technique.

\section{CONCLUSIONS}

We present in this work an accurate modeling based on multipole expansion of the Green's function integrating the STRD approach which allows to calculate the near generated by a rectangular micrometric aperture on parallel-plate waveguide. By considering a proper functionalization of the surface, the proposed layout can be applied for bio-sensing applications.

\section{REFERENCES}

[1] A. Massaro, L. Pierantoni, R. Cingolani, and T. Rozzi, "A New Analytical Model of Diffraction by 3D-Dielectric Corners," IEEE Tran. on Ant. and Propagat., vol. 57, no. 8, pp. 2323-2330, 2009.

[2] A Massaro, and R. Cingolani, "STRD and Near Field Modeling of Metallic Wedges for Optical Detection Systems," IEEE J. Light. Technol., vol. 28, no. 24, pp. 3562-3568, 2010.

[3] R. Marg, A. Mallwitz, and A. F. Jacob, "Radiation efficiency of apertures in parallel-plate waveguides," Microw. Opt. Technol. Let., vol. 21, no.3, pp. 159-162, 1999.L. R. Hirsch, A. M.Gobin, A. R. Lowery, F. Tam, R. A. Drezek, R. A., N. J. Halas, J. L. West "Metal nanoshells.," Ann. of Biomed. Eng., vol. 34, pp. 15-22, 2006.

[4] R. Shavit, "Impedance characteristics of a slot antenna fed by a parallelplate waveguide," Microw. Opt. Technol. Let., vol.14, no.2, 126-128, 1997. 